\title{
Error Analyses on English-language Public Signs in Shanghai Shopping Malls
}

\section{Yi Gao}

School of Naval Architecture, Ocean and Civil Engineering, Shanghai Jiao Tong University, Shanghai, China

\section{Email address:}

gaoyi229@sjtu.edu.cn

\section{To cite this article:}

Yi Gao. Error Analyses on English-language Public Signs in Shanghai Shopping Malls. International Journal of Applied Linguistics and Translation. Vol. 6, No. 1, 2020, pp. 29-41. doi: 10.11648/j.ijalt.20200601.15

Received: February 14, 2020; Accepted: March 10, 2020; Published: March 24, 2020

\begin{abstract}
Shanghai has recently undergone unprecedented transformation. With this level of international activity, the world expects Shanghai to be a model of standardized English-language public signs in China, and one would expect the city to apply a high standard to meet this expectation. However, this is not the case. English signs have the role of providing proper guidance for the international visiting public, but mistakes in usage defeat that purpose. This thesis focuses primarily on an error analysis of the English-language public signs in the most bustling and hustling commercial areas in Shanghai: Xujiahui, Huaihai Rd., Lujiazui, and West Nanjing Road. In addition to offering a collection of English-language translation errors from the major shopping malls of Shanghai, this thesis also investigates some of the errors in previous publications on English-language public-sign translation, which to a degree have misled the public for a long tisme. However, this thesis is not confined to error analyses using Nida's functional equivalence theory as a foundation; this thesis also proposes constructive strategies for avoiding future errors. The point of this thesis is to determine why translators make mistakes, and how they can avoid these mistakes. As such, this is a significant research with academic merit.
\end{abstract}

Keywords: English-language Public Signs Translation, Shanghai Shopping Malls, Error Analysis, Functional Equivalence Theory

\section{Introduction}

Significance of the Research

This thesis presents the current condition of the application of English signs in Shanghai shopping malls, as reported directly after the Shanghai World Expo pageant-selecting and analyzing errors in English-language signs in shopping malls through systematic and comprehensive investigation. Previous relevant studies are not systematic and specific; for instance, Error Correction Guide on English Public Signs mentions only a few examples of public sign translations in only certain places, with no systematic analysis of the mistakes or supporting theoretical background. Similarly, Guidelines for Chinese-English Translation in Public Places provides many criteria and translation theories for English signs in public places, but fails to point out common errors in translation and their causes. Thus, these works are incomprehensive, and not critical. This thesis applies Functional Equivalence Theory to discover and analyze the causes of these mistakes. Rather than mere error-correction, this thesis summarizes and analyzes the relevant problems.

Specifically, this thesis finds that errors in English-language public signs fall into three categories: linguistic errors, pragmatic errors, or cultural misunderstanding errors. These errors need to be treated differently. To resolve these issues, translators can draw support from Nida's functional equivalence theory, which says that it is very important to maintain the meaning of the source text to achieve the "closest natural equivalent" [11]. Therefore, the proposed strategies include borrowing the direct corresponding words or phrases from the target language, introducing isomorphs, and rewriting to avoid ambiguity to avoid ambiguity by using proper vocabulary and idiomatic expressions as often as possible.

In short, this thesis is focused on Shanghai, but its significance and application are not limited-the patterns revealed and principles proposed can be applied widely throughout China. 


\section{Functional Equivalence Theory as Applied to English-Language Public Signs}

Chinese-English Translation of Signs defines public signs as "the words or diagrams that publicly notify, instruct, remind, or alert the public regarding matters crucial to their daily life, productivity, environment, and business.”(公示语公开和面对公众，告示、指示、提示、显示、警示、标示 与其生活、生产、生命、生态、生业休戚相关的文字及图 形信息) [16]. Thus, English-language public signs in this thesis refers to the billboards, indicators, and slogans of shopping malls made in English, which present customers with both dynamic and static visual effectiveness. In short, the ultimate goal of these signs is to foster convenient shopping and transportation, and make customers feel at ease.

With this purpose, the following part will focus on the theoretical issues in discussing how functional equivalence theory can be applied in this field. Generally, functional equivalence theory includes four aspects: the equivalence of lexemes, syntax, discourse, and literary form, among which the total contained meaning is more crucial than any single aspect.

In Eugene Nida's theory, adequacy of translation is not confined to mere correspondence in lexicon and grammar between the source and target languages; the validity of a translation cannot stop with a comparison of corresponding lexical meanings, grammatical classes, and rhetorical devices [11]. Nida argues instead that translation should be regarded as a sort of communication between the readers and the translators [11]. How much readers can understand of the translation is determined by more than the lexical accuracy of the translated text, because the message delivered by the translation does not convey only lexical meaning at a superficial level but also hidden cultural information.

Therefore, reducing the deviation in the process of translation between different linguistic systems holds the balance in the adequacy of the translation. Nida calls it the "closest natural equivalent," the core of functional equivalence theory [11]. In other words, the closest natural equivalent implies a high degree of language-culture correspondence between the source and target languages, and an unusually effective translation allows the readers to experience something very close to what the original readers experienced.

Under the impact of economic globalization, a flood of Chinese products now swarm into international markets. Meanwhile, Chinese products featuring English translations and English-language public signs can be found in shopping malls throughout China. As such, applying functional equivalence theory in these translations to achieve the "closest natural equivalence" becomes a thoughtful and creative subject deserving of deliberation, as diverse cultures and cognitions are involved. Besides, keeping the correspondence in meaning between the target language and original text should be the priority of these translations.
Overall, a faithful translation will fit both the reader's language and culture in order to make the translated message intelligible and natural to target-language readers. In this regard, Nida's theory can serve as the guiding light in the development of public signs, and assist us to translate public signs to achieve better effectiveness.

\section{Mistranslations of English-Language Signs in Shanghai Shopping Malls}

This chapter turns to the pragmatic issue of collecting and analyzing mistakes in English-language public signs by applying Nida's theory.

\subsection{An Overview of Translation Errors}

Christine Nord defined translation error "as a failure to carry out the instruction implied in the translation brief, and as an inadequate solution to a translation problem" [6]. The scope of this research concerns the most celebrated and famous shopping centers in the most bustling and hustling commercial areas in Shanghai: Xujiahui, West Nanjing Road, Lujiazui and $\mathrm{Wu}$ Jiaochang. Apart from that, this research is also concerned with recent published findings and literature on the relevant topics.

In light of collecting the translation errors from all the shopping malls in these commercial areas, and reviewing the related literature on the topic, three typical types of translation errors become visible: linguistic errors, pragmatic errors, and cultural errors. The following sections employ Nida's functional equivalence theory and the relevant principles to comprehensively analyze such errors, and the reasons that people make these mistakes.

\subsection{Linguistic Translation Error Analysis}

Based on Nord's theory, linguistic translation error derives from inadequate translation when the focus is on language structures, leading to spelling, lexical, and grammatical errors [13].

\subsubsection{Spelling Errors}

In shopping centers, translation of public signage using correct English spelling is the basic requirement for translators. Proper spelling guarantees the fundamental purpose of preserving accurate and clear information. However, spelling mistakes on English-language public signs appear everywhere in Shanghai shopping malls-even in some famous European shopping centers in downtown areas. The following is a typical example:

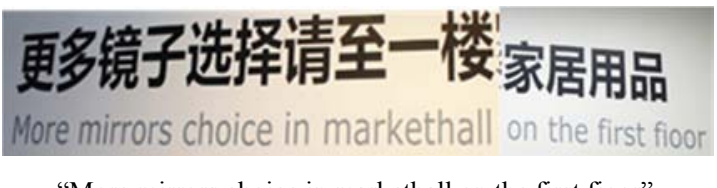

"More mirrors choice in markethall on the first fioor".

Figure 1. Spelling Errors. 
This is an English sign found in IKEA's Shanghai Xuhui Store-"floor" is misspelled as "fioor" and "market hall" as "markethall."

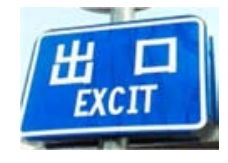

"Excit (出口)",

Figure 2. Spelling Errors.

In another typical spelling error, "Exit" is misspelled as "Excit." Based on Nord's analysis, these spelling mistakes seem to be caused by the carelessness of the translators. If more attention could be paid to spelling, most of these errors can be avoided.

\subsubsection{Grammatical Errors}

Another mistake stemming from the insufficient language competence of translators in grammar regarding how words are put together to make sentences [1]. Grammar indicates the language competence of human beings in expressing themselves, especially in applying structural foundation of the language. Grammatical errors occur when sentences counter the rules and conventions of the grammar-the morphology and syntax of the language.

For examples, we can first go back to Figure 1, above, which also contains a grammatical error: "mirrors choice". A noun adjunct (or attributive noun, or noun pre-modifier) is an optional noun that modifies another noun, meaning that it can be removed without changing the grammar of the sentence. However, noun adjuncts are traditionally mostly singular (e.g. "trouser press") except when there are lexical restrictions (e.g., "arms race"). In other words, the suggestion for "mirrors choice" should properly be "mirror choice" or "mirror choices", as "mirror" functions as a noun adjunct that cannot be presented in a plural form (and there are no lexical restrictions in this case). Moreover, the sign should not have omitted the preposition "for" in front of "more mirror choices," as it stands for the purpose of this pubic sign, which is to remind customers of the many other mirrors on the first floor.

Further, it's not an appropriately idiomatic expression, as an entire phrase cannot embody the meaning of Chinese character "请" in public signs. Further, based on the practical situation of IKEA's Xuhui Store, only one market hall contains two floors. Thus, it is unnecessary to mention "market hall" on the public signs. In short, the appropriate translation for this sign is, "For more mirrors, please go to the first floor."

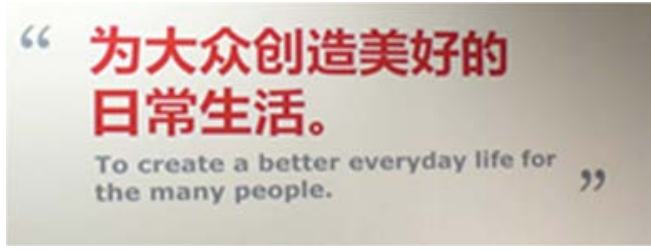

Figure 3. Grammatical Errors.

“To create a better everyday life for the many people (为大众创造美好的日 常生活).”
This sign also comes from IKEA's Xuhui Store. Obviously, the definite article "the" needs to be dropped. Thus, the modified phrase is "many people." However, "many people" is not the accurate expression for “大众," which simply means that the number of people is huge. The original Chinese phrase “大众” stands for the people in a country or community.

Nida's functional equivalence theory requires equivalence in lexemes. However, "no two words in any two languages are completely identical in meaning". Thus, "one purpose of translation is to keep such disparities at a minimum" [11]. In other words, the meaning of lexemes in the original language should correspond as closely as possible to the meaning in the target language. Finding a corresponding phrase in the target language for the original phrase is an effective way to reduce misunderstanding and distortion.

As noted, there are some phrases in two language that closely resemble to each other, such as "toilet" for “则所," “subway” for “地铁,” and “Taxi” for “出租.” We can borrow the corresponding phrases or words in the target language to translate what the original language wants to express. With these particular phrases, a translation can reach the effectiveness of functional equivalence. "The public" is the best translation for the Chinese phrase “大众."

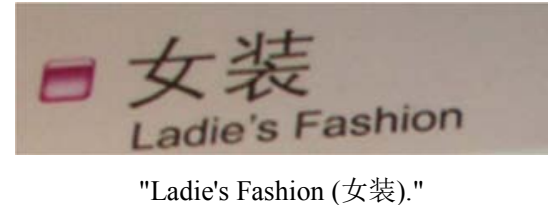

Figure 4. Grammatical Errors.

This sign from the BaiLian ShiMao International Plaza on West Nanjing Road commits a typical grammatical error: in the case of the plural possessive of nouns ending in "s," an apostrophe is needed at the end. Thus, the correct translation should be "Ladies' Fashion."

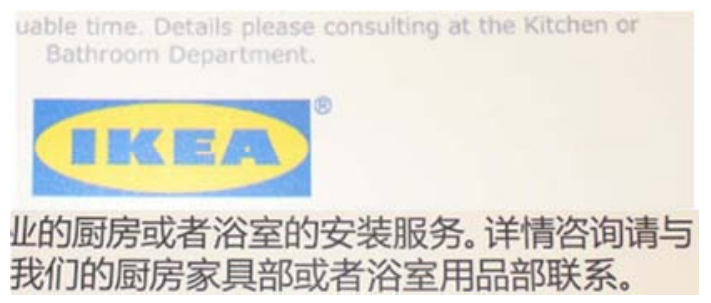

Figure 5. Grammatical Errors.

“Details please consulting at the Kitchen or Bathroom Department. (详情咨 询请与我们的厨房家具部或者浴室用品部联系).”

It's also from IKEA's Shanghai Xuhui Store. First of all, the word "consulting" should be replaced with its imperative verb form "consult," as there is a "please" before it indicating a request. Second, as with Figure 1, a modifier phrase cannot be just a noun, it is better to add the preposition "for" in front of "details". Third, there is also a lexical error, which is discussed in the following section.

After studying the five cases above, we see that grammatical errors are a little bit more complicated than 
spelling errors, as many grammatical rules are involved. However, on the other hand grammatical errors can be easily eliminated as long as we comply with the rules and conventions of the target language.

\subsubsection{Lexical Errors}

A lexical error is a deviation in form or meaning of a target-language's lexical word. Form deviations include orthographic or phonological deviations within the limits of single words, and also ignorance of syntactical restrictions resulting in false collocations, for example [5]. However, since deviations in form have been covered in the section on spelling errors, this section focuses mainly on deviations in meaning in a target-language and false collocation. Completely comprehending the phrases and words is essential prior to translation, as it determines what you want to express. But, merely paying attention to the meaning of words is not enough for a good translation. The proper application of collocations cannot be neglected, as it determines how accurate the translation is. Newmark defines a collocation "as the element of a system in the lexis of a language" [10]. The correct usage of collocation can secure the quality of the translation and make the sentence much more acceptable to target readers. The following examples illustrate the importance of collocations.

First, we need to revisit Figure 5 to solve the remaining issue concerning the phrase "Details please consulting at the Kitchen or Bathroom Department," regarding the collocation of "consulting at." According to the dictionary definition and the context of this sign, the verb "consult" is to ask for information or advice from someone who has special knowledge about a particular subject, and to discuss something with someone or get their permission before you make a decision. Thus, the relevant collocations are "consult somebody about something" or "consult with somebody" [7].

However, the Kitchen or Bathroom Department is the object of this verb in offering advice and suggestions for customers. Therefore, the correct collocation is to consult with the Kitchen or Bathroom Department. To make it concise, the preposition "with" could also be omitted. Therefore, the modified version for the English-language sign is, "For details, please consult the Kitchen or Bathroom Department."

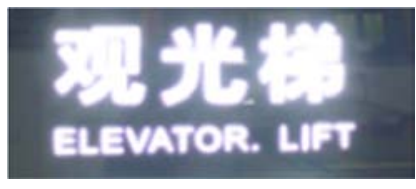

“'Elevator. Lift (观光梯)".

Figure 6. Lexical Errors.

This sign is taken in Orient Shop's Xujiahui Store.

It is obvious here that the translator misunderstood the meaning of "elevator," as it is identical to "lift." Therefore, the suggested translation for " 观光梯" is "sightseeing lift/elevator."

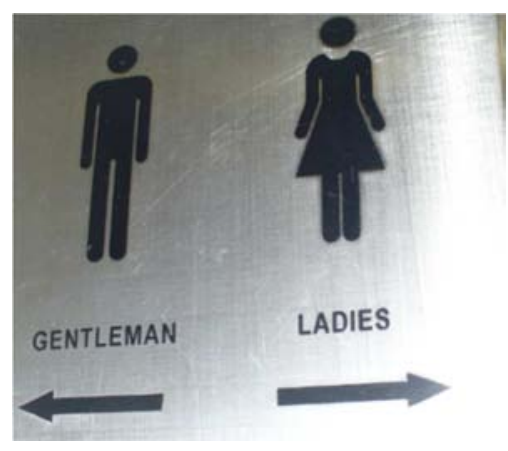

Figure 7. Lexical Errors.

This public sign is taken in Bailian Shimao International Plaza, West Nanjing Road. It is spelled correctly; however, "gentleman" and "ladies" fail to keep in accord with the nouns' number form-it is better to keep the same form, either in singular or plural. Thus, the suggested translation for this sign is either "gentlemen" and "ladies," or "gentleman" and "lady."

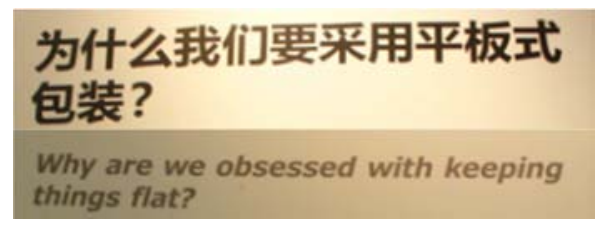

Figure 8. Lexical Errors.

“Why are we obsessed with keeping things flat? (为什么我们要采用平板式 包装? ). ”

There are multiple errors in this sign taken in IKEA's Xuhui Store. First, it's inappropriate to use the phrase "obsess with," as it indicates undue attention toward something or somebody, and is usually negative in its connotation. That is, people keep thinking about something, and find it difficult to think about anything else. It's too emotional to be used for this situation, which should be neutral and illustrative. Thus, the suggested correction is the word "use," "adopt," or "introduce."

In addition, this English-language sign also has another error-a pragmatic mistake that is discussed in the following section.

\subsection{Pragmatic Translation Error Analysis}

An error in pragmatic translation is the inappropriate expression of the translation or inaccurate comprehension of context to convey intentions. Most of the time, such errors will generate a so-called "pragmatic failure," a term first employed in 1983 by British linguist Jenny Thomas in Cross-Culture Pragmatic Failure, in reference to the failure to convey the intended message by one's words [15]. Similarly, the result of pragmatic failure in English-language public signs is that it makes the target readers struggle to understand what the original context intends to express.

Indeed, there is a flood of pragmatic errors in English-language public signs in Shanghai shopping centers. The consequences of such mistakes can be serious. Therefore, the causes and reasons for such errors must be noted and dealt with. There are several sub-types of this category, including 
ambiguities in the intended meaning, unidiomatic expressions, and distortions of meaning.

\subsubsection{Ambiguity in the Intended Meaning}

An ambiguity error refers to an expression of translation that goes against the intention of the original meaning in the source language and causes interpretations that confuse readers. Even though one of the features of public signs is conciseness which enables readers to catch the sign's meaning in a short time when the wording is not carefully crafted, ambiguity errors occur.

Going back to Figure 8, the phrase "keep things flat" does not completely translate the Chinese phrase "平板式包装." What's more, it gives viewers an incorrect impression that the IKEA staff are unaware of the process of packing, for they just keep products flat and throw the package to their customers. However, we know that all IKEA staff do care about their customers, which is contrary to what this English-language signage suggests. Actually, what the sign intends to tell customers is that keeping things flat is a smart way of packing, because it can save space and secure the safety of delivery. Thus, the suggested translation for this sign is, "Why do we introduce flat-packing?"

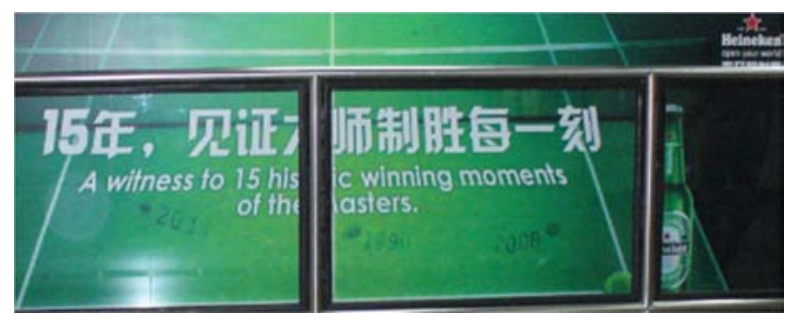

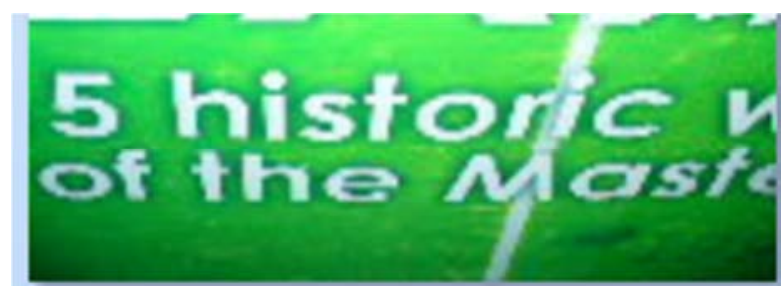

Figure 9. Ambiguity in the Intended Meaning.

“A witness to 15 historic winning moments of the masters. (15 年，见证大师 制胜每一刻).”

Photo taken in the underground passage leading to the Pacific Mall.

This is a public billboard made by world-famous beer company Heineken. From its English slogan, it's difficult to grasp the long history of success that the slogan wants to impress upon its viewers, because the word "moments" is too vague to convey the information that great masters have succeeded in making great beers for 15 years. "Moments" could be measured in seconds or minutes. If so, the English translation of this slogan could be conjured as "a great master gains his 15 personal successes in a very short time" which would be somewhat ridiculous. In order to avoid such an ambiguous interpretation, the suggestion is to give clear information for which moments quantify the "15 years." Moreover, "the great masters" is not a proper phrase, as "master" mentioned here is general instead of a specific group. Thus, the definite article "the" should be omitted. The modified phrase should be "great masters" or "a great master." To sum up, the suggested correction for this slogan goes as follows: "15 years, a witness to the winning moments of a great master!"

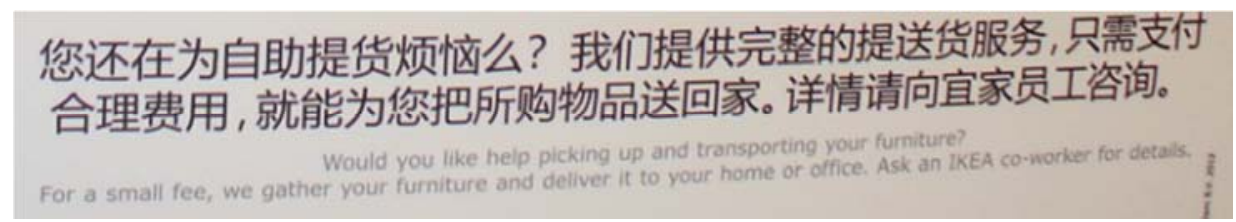

Figure 10. Ambiguity in the Intended Meaning.

"Would you like help picking up and transporting your furniture? For a small fee, we gather your furniture and deliver it to your home or office. Ask an IKEA co-worker for details. (您还在为自助提货烦恼么? 我们提供完整的提送货服务, 只需支付合理费用, 就能为您把所购物品送回家。详情请向宜家员 工咨询。）”

Photo Taken in IKEA's Xuhui Store

At the first glance of the English translation, the translator might have no idea of the implication and function of "Would you like..." It's an imperative sentence to ask others to do something for you. Therefore, the sentence "Would you like help picking up and transporting your furniture" could be rephrased as: "Please pick up and transport your furniture for me." Perhaps a better translation is, "Would you need help to pick up and deliver your furniture?" However, this still does not express the annoyance of customers with transporting furniture, which is stated in the original Chinese sign. Therefore, an even better translation would be, "Still annoyed with transporting furniture by yourself?" There is no doubt IKEA will provide the supporting service next when a reader sees this sentence.

Still, this translation is not perfect because "co-worker" stands for "colleague," so it's improper to use "co-worker" in this case. Moreover, "a reasonable charge" is different from "a small fee"; "a small fee" assumes that customers prefer to gain petty advantage, which is slightly insulting.

Taking all of this into account, the best translation should be this: "Still annoyed with transporting furniture by yourself? We'd like to help gather and deliver the furniture for you for a reasonable charge. For details, please consult the IKEA staff."

\subsubsection{Unidiomatic Expressions}

An unidiomatic expression, could be treated as a kind of interlanguage, which is an expression used by a learner of a 
second language who has not become fully proficient yet, but is approximating the target language. It's a kind of language use that violates the rules of the idiomatic expressions. Basically, it's English, but is deeply influenced by the characteristics of Chinese language and is therefore universally unreadable.

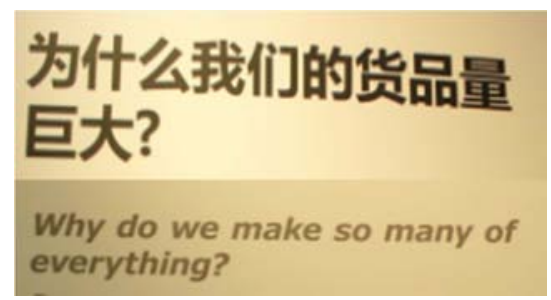

“Why do we make so many of everything? (为什么我们的货品量巨大? )"

Figure 11. Unidiomatic Expression.

\section{Taken in IKEA's Xuhui Store}

It's really hard to get what this English sign wants to express. However, the Chinese slogan shows that IKEA wants to impress its customers with the large varieties of commodities sold by IKEA. Thus, the suggested translation for this sign could be, "Why do we have such a large variety of goods?"

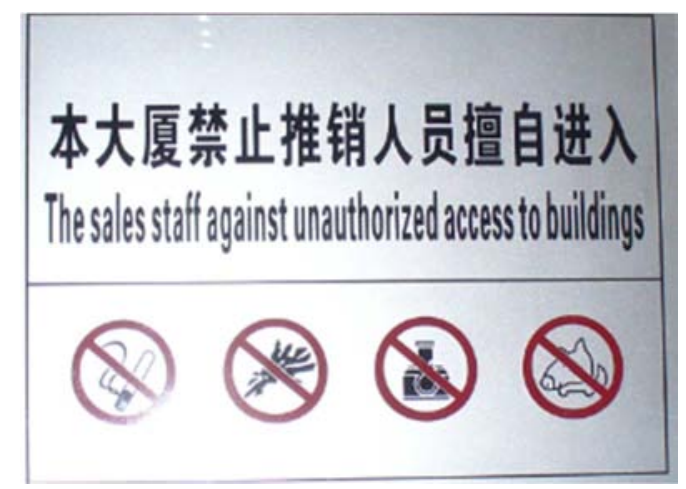

Figure 12. Unidiomatic Expression.

“The sales staff against unauthorized access to buildings 本大厦禁止推销人 员擅自进入"

Photo Taken in Shanghai Shangshi Mansion (North Caoxi Road)

This is an unidiomatic expression. One often sees public signs containing expressions of prohibition. In order to help people express them idiomatically, a summary of translations of prohibition expressions is included in Chinese-English Translation of Signs:

1. 1. No+ noun, for instance: “No food or beverage” (禁用 食物或饮料); “No tips” (不收小费)

2. No + doing, for instance: “No Fishing” (禁止钓鱼); “No Loading”(禁止装货作业)

3. No+ noun/doing + allowed, for instance: "No Ball Games Allowed" (严禁球类活动)

Compared with sample 1 and 2, the tone conveyed by sample 3 is much harsher, which prohibits the actions in a tougher manner [16].
Based on the above summary and combined with the intention of the sign, the sign's main purpose is to inform the public that no unauthorized person is allowed to enter this office building. The phrase "Staff Only, No Admittance" could generally express such an intention. However, the tone of this phrase is a little bit severe. The forbidden subject is not specific according to the Chinese sign, which in fact specifically does not allow an unauthorized salesman to enter. Therefore, the usage of "No + noun" is appropriately applied: "No Access for Salesmen." But, this still seems to be not fully concise, as "access" is redundant. Because the notice is on the main entrance of this building, even without "access" no one would become confused. Thus, the suggested translation for this Chinese sign is simply "No Salesmen."

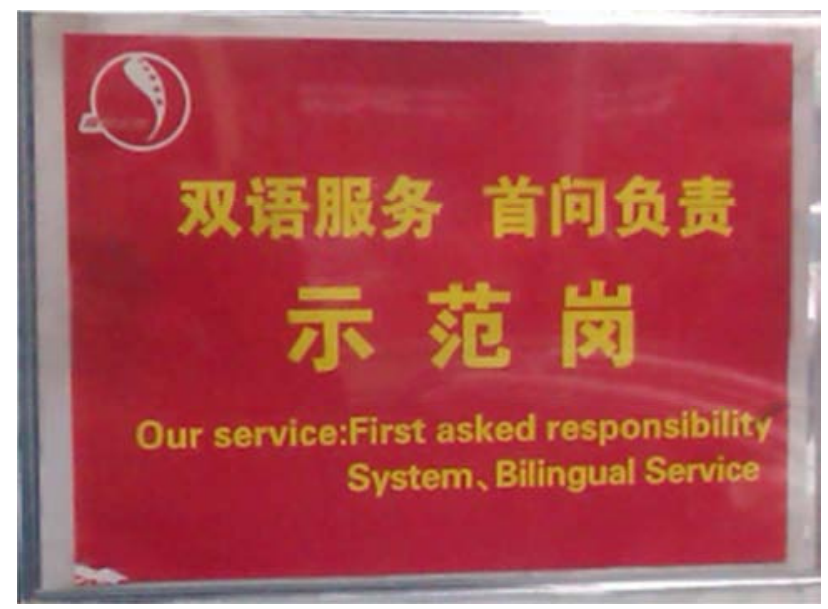

Figure 13. Unidiomatic Expression.

“Our Service: First asked responsibility System, Bilingual Service （双语服 务首问负责 示范岗) ”

Taken in Shanghai South Bus Station

In functional equivalence, Nida set a bottom-line for translation, saying that "readers of a translated text should be able to comprehend it to the point where they can conceive of how the original readers of the untranslated text must have understood and appreciated it" [11],

Figure 13 is a typical mistake in this regard, with word-for-word translation and Chinese features that often turn out to mean nothing. For instance, no readers of the translated text would catch the intended idea simply from "First asked responsibility System" and "Bilingual Service," because these two phrases are too vague to be understood. How should a reader even interpret "first asked"? Why is "bilingual service" involved? What is the relationship between the readers of the translated text and the "system"? Further, the Chinese phrase “示范岗”is simply omitted in the translation. Apparently, this English-language notice could not offer a satisfactory translation. No doubt the target readers find it very difficult to conceive how the original readers of the text understand and appreciate it.

Nida's functional equivalence proposes that if the original text presupposes considerably more knowledge about the contents than can be expected of the readers of the translation, 
then this may require a type of "rewrite" so as to make the text of the translation acceptable to readers [11]. In other words, it's a kind of "free" translation using words and phrases of the translated language to paraphrase the connotation or implied meaning of the original text.

For instance: "He thinks by infection, catching an opinion like a cold."

\section{人家怎么想他就怎么想,就像人家得了伤风,他就染上感} 冒[6]。

Looking at this translation, the implied connotation of the original text cannot be conceived simply by the superficial word-for-word translation, as the implied meaning is hidden between the words. Thus, it requires a pair of keen eyes to uncover the hidden message and bring it out so that the readers of the translated text can appreciate what the original readers understand.

Back to this Chinese notice-in fact, it presents a strong air of Chinese culture and word characteristics. Due to the huge differences between the two languages systems, simply applying a word-for-word translation will lead to considerable confusion.

In the light of Nida's theory, the major error is that the translator neglects the implied content of the Chinese phrases “首问负责” “示范岗.” The implication of the phrase “双语 服务，首问负责” means that whenever a customer makes an inquiry, whoever is in charge of the customer support at the time will take the inquiry and be responsible for providing a satisfactory solution in a short time. Further, the language used in the service is not confined to Chinese, and other languages are available. Therefore, the primary intention of the sign is to offer the major implication of this system, rather than giving the literal meaning of it; readers of the translated texts need the implied content of this sign and an understanding of what kind of help they can get, rather than the official name of the system. Generally speaking, the sign is for people who have problems in the station and need help from staff-thus, the corresponding translation is "Guaranteed satisfactory solution with bilingual service." This gives a straightforward meaning for people: if you have any problem about your travel, you can come here and get a satisfactory answer in your own language. Moreover, the staff member you first inquired with will take responsibility to answer in a short time.

Moreover, the other phrase “示范岗” is omitted in the translation of English sign, which is not proper. In reality, an information desk or service center is present in the station to provide service for passengers. In this case, this information desk is regarded as a model to provide excellent service. Thus, the suggested translation is "model service center" or "model information desk."

To sum up, the suggested translation for the entire slogan is then "Model Service Center: Guaranteed Satisfactory Solution with Bilingual Service."

\subsubsection{Distortion of Meaning}

Distortion of meaning occurs when the translation does not match the source language and hence distorts what it means.
Following are two examples of this type of mistake.

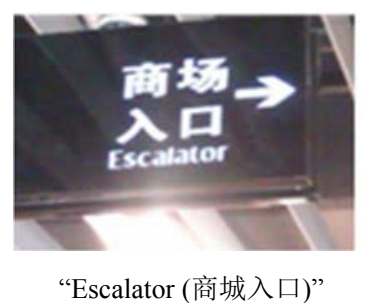

Figure 14. Distortion of Meaning.

Photo Taken in Next Age Shopping Center

This is the public sign on the ground floor in the Next Age Shopping Center (Yaohan store, Lujiazui). At first glance, the English translation does not match the Chinese version. Actually, an elevator does indeed exist if a customer follows the direction of this sign. But, what if a customer wants to find the entrance to the shopping center? Can the English word "escalator" make any sense without an indication of the entrance? Therefore, "Escalator to the Mall" shall be a better translation.

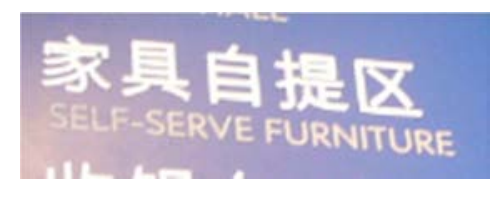

“Self-Serve Furniture (家具自提区)”

Figure 15. Distortion of Meaning.

Photo taken in IKEA's Xuhui Store

This sign comes from IKEA's Xuhui Store. "Self-Serve Furniture" seems to tell the customers that IKEA has some sort of intelligent furniture that can provide multifunctional services for human beings or even serve itself. However, if an English-speaking customer becomes curious about this innovative and magic furniture and follows the sign, he will eventually be disappointed. Based on the Chinese sign, it indicates customers can pick up products on their own from the warehouse. Therefore, the correct translation for this sign should be "Self-Serve Warehouse."

\subsection{Cultural Misunderstanding Error Analysis}

Culture is defined as "the totality of beliefs and practices of a society" [9]. Therefore, closely related with culture, language is endowed with a social function. To translate different languages is to engage in cross-cultural communication. Hence, translation can also be regarded as a part of culture.

Nida believes that bilingual competence has almost always been regarded as an essential requirement for translators, but this does not always mean that one must have an active competence in both the source and target languages. For a truly successful translation, biculturalism is even more important than bilingualism, since words have meanings only in terms of the cultures in which they function [11]. Therefore, it's indispensable to understand the implied cultural meaning of the target language when translation is processed. 
Nida also notes that some of the difficulties of translation stem from culturally idiosyncratic meanings [11]. Hence, it's not surprising that the distorted implications in some unacceptable public translations actually derive from an unawareness of the culturally idiosyncratic meaning. Nevertheless, some cultural translation errors still exist with twisted roots to mislead the reader. For instance, the differences among "parking," "car park," and "parking lot" have been confusing people for a long time. Even some public-sign translation guidelines published by government officials do not avoid such mistakes. Now, we turn to this kind of cultural mistranslation.

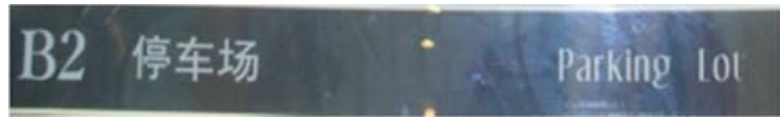

“Parking Lot (停车场)"

Figure 16. Distortion of Meaning.

Photo taken in Next Age Shopping Center (Yaohan store, Lujiazui)

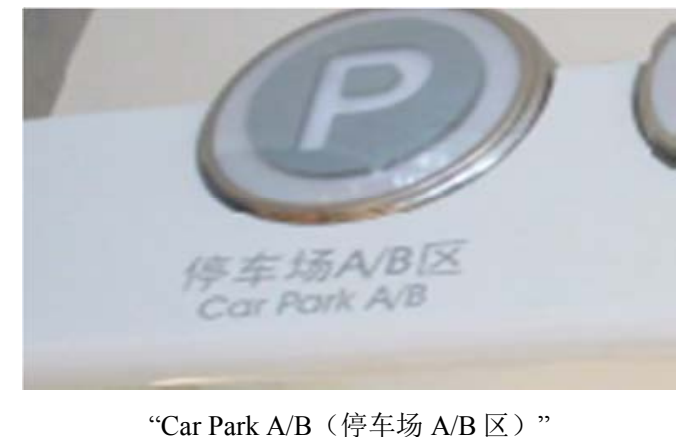

Figure 17. Distortion of Meaning.

Taken in Shine Time Shopping Center, Shanghai South Railway Station

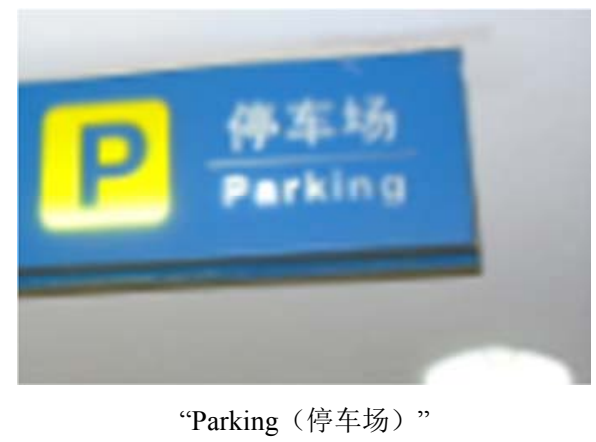

Figure 18. Distortion of Meaning.

Photo Taken in New World Shopping Center, West Nanjing Road

With respect to the translation of "停车场," there are many versions. Among them, "Parking Lot," "Car Park," and "Parking" (Figures 16, 17, and 18) are the three most-used. But, which one is correct? Are they equally valid? Indeed, this issue has triggered great interest and controversy in the field of translation. Many resources focusing on public-sign translation hold different views.

Wang Xin and Lü Tingting argue that "Parking" is for "停 车场," while "Reserved Parking" matches "专用停车位." "Car Park" is also another expression for "停车场," which can often be seen in English-speaking countries [17]. A Companion to the English Translation of Public Signs, by the Jiangsu Province Language Work Committee Office, concludes that the word "停车场" has three different English expressions-"Parking," "Car Park," and "P,"-while "Parking Space" is for "停车车位" [4]. Chinese-English Translation of Signs, whose authors undertook field investigation in London on English-language public signs, says that "Car Park" is for " 停车场" [16]. However, The Error Analysis and Criterion of English Signs in Public Signs, composed by the Shanghai Language Word Committee Office, prefers "Parking (Lot)" for "停车场," and "Parking Space" and "Parking Stall" for " 车位" [14]. Overall, the translation of "停车场" differs from book to book, but none of them offers a meaningful explanation for any specific translation.

Nevertheless, from the authoritative Macmillan English Dictionary we know that "parking" has two meanings: one is the process of putting a vehicle into a place (such as a parking space) and leaving it there, and the other is a space itself where motor vehicles can be left [7]. We might still ponder whether "parking" can serve as a standard expression for the translation of "停车场." This dictionary doesn't provide us with a definite answer, but it does give two expressions for "停车场 "-"parking lot" and "car park." The dictionary further clarifies that "parking lot" refers to an area or building where people can park their cars and is exclusive to American English, while "car park" is the standard in British English.

Now we are quite sure that "Car Park" and "Parking Lot" are both standard expressions for the translation of "停车场." However, the usage of "Parking" still remains unexplained. Collins Cobuild Advanced Learner's English-Chinese Dictionary provides us with an explicit idea on the translation of "Parking." Even though it agrees with the definitions of "Parking" in the Macmillan English Dictionary in general, it points out that "Parking" is "the space for parking a vehicle in," with a clear corresponding Chinese expression "停车位" [1]. It also offers the specific explanation of the usage of the related words: you do not use the word "parking" to refer to a place where cars are parked. Instead, you talk about a car park in British English or a parking lot in American English. "Parking" is used to refer only to the action of parking your car, or to state of being parked... hence, a "No Parking" sign [1].

Through the above analysis, the differences among these phrases become clear. "Parking” in Chinese means “停车位” rather than “停车场," especially when it's used alone. There are several expressions for “停车位, such as "Parking Stall” and "Parking Space." The translations of “停车场” are "Parking Lot" and "Car Park," representing different cultural backgrounds-if the translation of signs generally applies British style but one particular sign uses American style, then this is improper. 


\section{Translation Strategies for English Signs in Shanghai Shopping Malls}

English-language public signs serve as the face of a city, and play a very important role in shaping China's international image especially in a major metropolitan city like Shanghai. The adequacy of English-language public signs also indicates the development of the city's internationalization. However, this study has shown that errors in English signs are everywhere in our daily life. This situation needs to be changed, and this chapter suggests several strategies for avoiding such mistakes. To deal with the problem of English-language public signs, we must seek assistance from translation theories and related guidelines for English-language signs. Thus, the following sections discuss the relevant strategies for avoiding this.

\subsection{Borrowing the Directly Corresponding Words or Phrases from the Target Language}

In translation using Nida's functional equivalence, correspondence in meaning is the first priority in the process of translation. Ideally, the translation could serve as a mirror for the original text. In fact, some words or phrases actually have direct corresponding translations in the target language. Nida proposes that if a more-or-less literal correspondence is functionally equivalent in both designative and associative meaning, then no adjustments in form are necessary [11]. In other words, literal correspondence is a translation strategy to preserve both the content and the form of the original text at the same time. Further, it's often easily accepted and appreciated by target readers.

In terms specifically of translation into English-language public signs, a literal translation refers to words or phrases in the original text that possess almost an exact correspondence in the target language-both share the same designative and associative meaning, and are functionally equivalent. Applying the strategy of literal translation not only reduces misunderstanding and distortion within two different language systems, but also preserves local features.

For instance, in Figure 3 from the previous chapter, "the public" corresponds directly to the Chinese phrase "大众"; In Figure 18, "car park" and "parking lot" both directly match “停车位." Although "car park" is the expression in British English and "parking lot" is exclusive to American English, the phrases are the direct corresponding translation of the original text. They keep the approximately-identical implied meaning and social function, and can be regarded as the closest natural equivalence.

\subsection{The Introduction of Isomorphs}

However, such a close correspondence is not always available. Isomorphs can be used when the implied meaning and culture cannot be expressed concurrently in the translation, and the literal translation does not help.

Under Nida's theory, there are usually no exactly-corresponding words or phrases in two different languages, due to the differences existing between the two language systems and cultures [11]. Thus, he proposes the theory of "isomorphs" to decrease the loss and distortion in translation; Nida regards this as a useful way to achieve functional equivalence: "We may define functional isomorphs on the basis of the means for accomplishing essentially the same results within different systems. This means that for the English idiom to grow like mushrooms it is appropriate in Chinese to talk about growing like bamboo shoots" [11]. Therefore, the English idiom "to grow like mushrooms" is the isomorph of the Chinese idiom "to grow like bamboo shoots."

In other words, the functional isomorphs indicates the signifier and signified of two symbols within different language systems that are closely similar to each other much like an accurate landscape painting is similar in appearance to reality. Such a strategy is very helpful in the translation of English-language public signs, especially in dealing with the lack of direct corresponding words or phrases.

Take Figure 15, for instance. The Chinese phrase “家具自 提区" refers to the place storing goods like furniture where customers can pick up products by themselves. The word “区” in English could have several different meanings: district, area, region, or sector. However, due to language differences and cultural background, such translations do not present an accurate interpretation of what the signified meaning of the original text conveys. Moreover, people of the target language are likely have no concept of "区" in the cognitive world of their language, as the notion "区" is a very Chinese characteristic.

Thus, such a translation needs help from the strategy of "functional isomorphs," which is to find the functional word in the target language for this particular meaning of the original Chinese word “区." In fact, people from English-speaking countries often use "warehouse" to refer to the place where goods like furniture are stored. This word meets the function of "区" in the original text. Thus, "warehouse" is the isomorphs of "区" in the translated text. Though they differ in their separate semantic meanings and structures in many aspects, they nonetheless both express the functional equivalence of the place where goods are stored-just like "mushroom" and "bamboo shoots," both of which describe the rapid growth of something.

\subsection{The Application of Rewriting}

If the translation strategy of "isomorphs" still fails to convey the connotation and culture of the original text, then the application of rewriting is a useful way to achieve functional equivalence in meaning between two different languages. Nida argues that if the original text presupposes considerably more knowledge about the contents than can be expected of the readers of the translation, then it may require a type of "rewrite" so as to make the text of the translation acceptable to readers [11]. This refers to the transfer of the deep structure of the original language to the surface structure of the target language [3], by interpreting the connotation of the original language in the words and phrases of the target language. 
The translation of “首问负责” in Figure 13 is one of the cases for applying the strategy of rewriting as it contains local cultural background and strong features of Chinese; Thus, the only method left is to reconstruct the original text and uncover the implied meaning by applying the words and phrases of the target language. Therefore, the corresponding translation is "Guaranteed satisfactory solution".

\subsection{Avoiding the Ambiguity of the Translation}

The function of English-language public signs in shopping malls is to inform, promote, and prohibit the activity of international customers. Thus, it is a priority to keep these signs clear, simple, and straightforward for readers. Nida argues that "meaning must be given priority, for it is the content of the message which is of prime importance" [12]. English shopping signs, however, differ from other genres in terms of style and rhetoric, and so it's equally important during the process of translation to maintain the meaning of the source text while also avoiding ambiguity.

According to Nida, translation ambiguity arises from these factors as follows:

"Obscurity of meaning may have several causes: the intent of the source (this is often the case in political statements and esoteric documents), orthographic corruption, and inadequate dictionary or encyclopedic information so as to reconstruct the context of the documents in question" [11].

In light of Nida's theory, causes of errors in English-language public signs include the intent of the source and inadequate dictionary or encyclopedic information. Similarly, without referring to the dictionary or encyclopedia, translators misused the phrase "would you like" in Figure 10. Apart from that, ambiguity caused by syntax also cannot be ignored-the billboard of "a witness to 15 historic winning moments of the masters" in Figure 9, and the notice of "the sales staff against unauthorized access to buildings" are good examples to demonstrate the necessity of accurate grammar and sentence structure.

Further, Nida's theory also provides us with a prescription for avoiding ambiguity in the production of functional equivalence. He says,

"If a close formal translation is likely to result in a misunderstanding of the designative meaning, (a) certain changes must be introduced into the text of the translation or (b) the literal translation may be retained and a footnote explaining the likely misunderstanding must be added" [11].

In terms of English-sign translation, the primary goals are that it shall be clear, short, and brief; therefore, adding a footnote explaining the likely misunderstanding is not appropriate for the translation in English signs-customers take only a short time to read the signs when passing by. However, Nida's suggestion (a) is valid and applicable. These so-called "certain changes" to avoid possible misunderstanding of designative meaning are primarily lexical, but also involve matters of syntax [11].

With examples like "In case of fire please use the lift," it's simple enough to replace the "in case" with "in the case"-the ambiguity of the English sign suddenly disappear. Similarly, the notice "The sales against unauthorized access to buildings" can be replaced by a simple few words, like "No Unauthorized Salesmen"; its chief idea is easily caught by readers.

The above sections have involved the discussion of strategies to resolve errors mainly from lexical misuse, in both the linguistic and pragmatic areas. Useful strategies include the application of direct corresponding words or phrases from the target language, the introduction of isomorphs, and (when necessary) rewriting to avoid ambiguity in translation. The following sections will try to solve the errors mainly caused by lack of familiarity with a language or culture.

\subsection{Improving a Translator's Attitude and Competence}

The most basic goal of a good translation is to spell correctly, and the main cause of misspelling is simply the carelessness and negligence of translators. They need to realize the importance of correct spelling, and to pay more attention to it. This should not be a serious problem because they can use spell-check functions, online resources, or dictionaries. Indeed, there is no excuse for misspelling when multiple tools are available. As long as translators treat their project with a careful and serious attitude, this type of mistake could be completely eliminated.

Apart from spelling, translation comes down to the right usage of words and the proper competence in mastering English grammar. As Zhang Jian argues that the language competence of a translator is of vital importance. Zhang also points out some useful strategies at the linguistic level, which are:

"To master the comprehensive language knowledge on the one hand and to possess a large pool of vocabulary on the other hand, both are needed. Merely having grammatical knowledge without sufficient vocabulary, the translator has to consult a dictionary constantly, which will greatly reduce the translation speed and affect the accuracy of the translation. However, without decent grammar and rhetoric, even if the translator possesses a large pool of vocabulary and thoroughly comprehends the source text, he or she still could not come up with a well-organized translation, let alone a decent and authentic interpretation" [18].

(一方面要掌握全面的语法知识, 另一方面要有很大的 词汇量, 两者缺一不可。光有一点语法知识, 没有一定的 词汇量, 这就会迫使译者不断地查阅双语词典, 打断正常 的思路, 这既大大地降低了翻译的速度, 又影响了翻译的 准确性。如果只有大量的词汇, 而没有较好的语法和修辞 知识, 那么在报刊翻译中, 译者尽管理解了原文, 却 无 法组织像样的译文, 更谈不上地道的译文。)

For instance, if a translator has thorough competence within both languages systems, no spelling errors like "fioor," "markethall," and "excit" will appear (as in Figures 1 and 2). Similarly, there will be fewer wrong collocations like "consult at the Kitchen or Bathroom Department" in Figure 5. Language competence prevents such errors.

To sum up, improving the grammar skills of translators and enlarging their vocabulary are very essential during the process of translation. Only when this has been done can translators improve their competence and produce an acceptable English translation for public signs. 


\subsection{Introducing an Out-Review Process}

Introducing an out-review process would also help to secure the quality of the translation. This process refers to the third party review: sending drafts to well-established experts in the field to seek revision advice for public-sign translations.

Indeed, it's very difficult for a public-sign translator to recognize some of the errors that can exist in his or her translation. As Zhang Jian argues,

"A translator's language competence is the vital element in determining the quality of the translation. A text that a translator can interpret right now might have been too difficult for him to understand 10 years ago; similarly, a translation interpreted by a translator 10 year ago might have to be re-done right now" [18].

(译者的语言修养程度是决定译品优劣的重要因素。一 个译者现在能译的东西, 十年前可能读都读不懂; 十年前 译的东西, 现在可能会推到重译.)

This tells us that the language competence of a translator undergoes changes objectively. High translation competence requires endless pursuit of personal improvement in language learning. However, there perhaps exists a shortcut. If there happens to be another translator who possesses the knowledge that you lack, then unnecessary translation errors can be avoided by consulting that translator. This points to an out-review process to secure the quality of translations of public signs.

In addition, since the errors of English public signs discussed here are a matter not only of translation quality, but also of the public image of the city itself, Shanghai perhaps needs to impose a supervisory system on English-language public signs to secure their quality.

\subsection{Strategies for Cultural Translation}

Given the related samples mentioned in the section on cultural misunderstanding in error analysis, it's not hard to conclude that most errors lie in the inadequate exploration of background cultures, and in an ignorance of etymology.

Nida concludes that bilingual competence has almost always been regarded as an essential requirement for translators, but this does not always mean that one will actually have an active competence in both the source and target languages. However, for a truly successful translating, biculturalism is even more important than bilingualism, since words have meaning only in terms of the cultures in which they function [11].

Having good command of two languages does not guarantee a good translation; however, only when translators are capable of valuing the important role played by the cultural and social backgrounds behind the source text can they present a faithful translation for their readers. Hence, translators must be fully aware of cultural differences and background when working with the source text. In particular, referring to different dictionaries and making full use of internet resources can help translators to find the sources of the words and their functions in modern culture.

Further, translations cannot neglect the understanding of intended readers. Thus, the review process mentioned earlier should be reiterated here: all drafts of translations should be reviewed from the perspective of target readers. Beyond that, it's simply better to have a second review by native language speakers who are good at translation. With this, misunderstandings of culture and social areas can be avoided.

\subsection{Other Suggestions}

\subsubsection{The Proper Usage of Pinyin}

This section concerns the proper usage of Pinyin in the translation of English-language public signs. "Han Yu Pin Yin" is regarded as the best component for the Chinese transliteration of words during the process of translation [2].

Basically, there are two types of translation in the process of translation from Chinese:

a) The transliteration of a Chinese untranslatable word

A Chinese untranslatable word or phrase might have different meanings and rich connotations. It can be difficult or impossible to express local culture, customs, and legends in free translation. For instance:

城隍庙 Chenghuang Temple

臭豆腐 Chou dou fu

龙华寺 Longhua Temple

b) The translation of local places in China

The proposal of employing Han Yu Pin Yin as the standardized Romanization of Chinese geographical names was put forward by the Chinese government and approved during the Third Conference of the United Nations on the Standardization of Geographical Names in 1977. That is to say, we now agree to employ Pinyin to translate local places in China-for instance,

剑川路 Jianchuan Road

南京西路 West Nanjing Road

\subsubsection{Avoiding Discrimination in English-Language Public Signs}

A century ago, there was a sign declaring, "Chinese and dogs are forbidden" in the Bund. Such a humiliating sign imprinted our Chinese consciousness. Since then, we have aspired for freedom from discrimination all over the world. Unfortunately, some discriminational English-language public signs still exist in Shanghai, the result of a lack of prudence in our Chinese translators.

Let's focus on the modified Figure 12, "No Salesmen"(本 大厦禁止推销人员擅自进入). This building does not allow salesmen to enter, which implies personal discrimination. "Salesmen" refers to people occupied in the sales profession. Such a professional should enjoy the right of access to a building, along with everyone else. Any notice of restriction based on his professional identity is a kind of personal discrimination. In this sign, it is the activity of sales promotion that should be forbidden, instead of the salesman.

Due to China's international standing, any kind of discrimination on public signs will leave a bad impression on tourists visiting Shanghai. The suggested strategy is to have innocuous notices that focus on a certain activity rather than a personal identity. This avoids personal discriminations on 
public signs. Therefore, "No Salesmen" shall be replaced with "No Selling Activities."

\section{Conclusion}

English-language public signs in Shanghai shopping malls, an important part of the linguistic and cultural environment, play a critical role in this international metropolis. Any misuse of language will undoubtedly leave a bad impression on the public, and even cause misunderstandings.

As such, this thesis focuses on the error analysis of English translations on public signs in Shanghai shopping malls, including billboards, indicators, instructions, notices, and slogans. Moreover, this thesis also provides several strategies for resolving such problems based on functional equivalence and other theories.

To sum up, this thesis finds that errors in English-language public signs can be categorized as one of three types: linguistic errors, pragmatic errors, or cultural misunderstanding errors. Linguistic errors fall into two major categories, lexical errors and grammatical errors. Lexical errors include deviations in form (spelling errors) and false collocations or meanings of a target-language lexical word. Pragmatic errors refer to ambiguity of intended meaning, distortion of meaning, and unidiomatic expressions caused by-word-for word translation. Cultural misunderstanding errors derive from misunderstandings between different cultural backgrounds, and specifically from a neglect of the history of vocabulary usage.

Each of these errors is dealt with differently. Errors in both the linguistic area and pragmatic area result primarily from deviations in meaning, and are the dominant errors on English-language public signs. To resolve these issues, translators can draw support from Nida's functional equivalence theory, in which it is very important to maintain the meaning of the source text to achieve the "closest natural equivalent" and to avoid ambiguity by using proper vocabulary and idiomatic expressions as often as possible. Strategies include borrowing the direct corresponding words or phrases from the target language, introducing isomorphs, and rewriting to avoid ambiguity.

Beyond improving the translator's language competence and quality of biculturalism, an effective strategy is to establish a second review process to have the translation reviewed by native translators (or at least native speakers) of the target language. This can be crucial in eliminating errors from spelling, grammar, and cultural misunderstanding.

Further, applying the proper usage of Pinyin and avoiding discrimination in English-language public signs are also important suggestions that deserve attention in translation techniques.

Errors in English-language public signs are an important matter for Shanghai's public image-serious attention and additional resources should be invested in this field. This thesis is my first attempt to address the problem. However, due to the depth of Nida's functional equivalence theory and the large breadth of errors in English-language public signs, this thesis alone cannot nearly hope to resolve the issue. No doubt more research will be done, and more investigation of related theories, in order to advance this field.

Hopefully, through the constant efforts of researchers, a system that guarantees the correct usage of English signs in Shanghai will be established, and the current errors in public areas will be addressed. With our efforts, Shanghai-the window of China to the world-can become the model of proper usage of English-language public signs.

\section{References}

[1] Collins Cobuild Advanced Learner's English-Chinese Dictionary [M]. Beijing: Foreign Language Teaching and Research Press, 2011: 1155-1903.

[2] Cui Xuexin. Guidelines for Chinese-English Translation in Public Places [M]. Hangzhou: Zhejiang University Press, 2010: 2-4.

[3] Guo Jianzhong. Contemporary Translation Studies in USA [M]. Wuhan: Hubei Education Press, 2000: 67.

[4] Jiangsu Language Work Committee Office. A Companion to the English Translation of Public Signs [M]. Nanjing: Nanjing University Press, 2010: 148.

[5] Liach, María Pilar Agustín. Lexical Errors and Accuracy in Foreign Language Writing [M]. UK: Multilingual Matters, 2001: 75 .

[6] Liu Miqing. Style and Translation (revised version) [M]. Beijing: China Translation \& Publishing Corporation, 1998: 122.

[7] Macmillan English Dictionary [M]. Beijing: Foreign Language Teaching and Research Press, 2003: 293-1013.

[8] Méjri, Salah. Traduire La Langue, Traduire La Culture [M]. Paris: Maisonneuve et Larose, 2003: 193.

[9] Newmark, Peter. Approaches to Translation [M]. Shanghai: Shanghai Foreign Language Education Press, 2001: 113.

[10] Nida, Eugene A. Language Culture and Translating [M]. Inner Mongolia: Inner Mongolia University Press, 2001: 236328.

[11] Nida, Eugene A. and Charles R. Taber. The Theory and Practice of Translation [M]. Leiden: E. J. Brill, 1982: 13.

[12] Nord, Christine. Translating as a Purposeful Activity-Functionalist Approaches Explained [M]. Shanghai: Shanghai Foreign Language Education Press, 2006: 75.

[13] Shanghai Language Word Committee Office. The Error Analysis and Criterion of English Signs in Public Signs [M]. Shanghai: Shanghai Foreign Language Education Press, 2010: 3.

[14] Thomas, Jenny. "Cross-Cultural Pragmatic Failure" [J]. Applied Linguistics, (1983) 42: 91-112. Accessed February 20, 2013, doi: 10.1093/applin/4.2.91.

[15] Wang Ying. and Lü Hefa. Chinese-English Translation of Signs [M]. Beijing: China Translation \& Publishing Corporation, 2007: 13-33, 344. 
[16] Wang Xin et al.. Error Correction Guide on English-language Public Signs [M]. Beijing: China Book Publishing House, 2010: 72 .
[17] Zhang Jian. Newspaper Language Translation [M]. Beijign: Higher Education Press, 2008: 37. 